\title{
PERMUTABLE POWER SERIES AND REGULAR ITERATION
}

\author{
I. N. BAKER \\ (received 3 August 1960)
}

\section{Introduction}

If $f(z)$ is a power series

$$
f(z)=\sum_{n=1}^{\infty} a_{n} z^{n}
$$

convergent for $|z|<\rho$, where $\rho>0$, then $f(z)$ is said to have a fixpoint of multiplier $a_{1}$ at $z=0$. In the (local) iteration of $f(z)$ one studies the sequence $\left\{f_{n}(z)\right\}, n=0,1,2, \cdots$ in a neighbourhood of $z=0, f_{n}(z)$ being defined by

$$
f_{0}(z)=z, f_{n}(z)=f\left\{f_{n-1}(z)\right\} \text { for } n=1,2,3, \cdots
$$

For many values of the multiplier $a_{1}$, including $0<\left|a_{1}\right|<1$ and $\left|a_{1}\right|>1$, the local iteration of $f(z)$ is completely mastered by the introduction of Schröder's functional equation

$$
\chi\{f(z)\}=a_{1} \chi(z) .
$$

Koenigs [11] showed that, if $0<\left|a_{1}\right|<1$ or if $\left|a_{1}\right|>1$, there is a unique function $\chi(z)$ which satisfies $(S)$ and has an expansion

$$
\chi(z)=z+\sum_{n=2}^{\infty} b_{n} z^{n}
$$

convergent in some neighbourhood of $z=0$. Any other solution of (S) which is regular at $z=0$ is a constant multiple of $\chi(z)$. Not only can one express all the "natural" iterates $f_{n}(z)$ as

$$
f_{n}(z)=\chi_{-1}\left\{a_{1}^{n} \chi(z)\right\}
$$

in suitable neighbourhoods of $z=0$, but with arbitrary real or complex $\lambda$ and suitable determinations of $\boldsymbol{a}_{1}^{\lambda}$ the expression

$$
f_{\lambda}(z)=\chi_{-1}\left\{a_{1}^{\lambda} \chi(z)\right\}
$$

generalises $f_{n}(z)$ to a family of continuous iterates $f_{\lambda}(z)$ which are permutable in the sense 


$$
f_{\lambda}\left\{f_{\mu}(z)\right\}=f_{\mu}\left\{f_{\lambda}(z)\right\}=f_{\lambda+\mu}(z) .
$$

The same calculations may be carried out formally in the case $a_{1}=\exp (i \theta)$ where $\theta$ is real and irrational. Cremer [4] and Siegel [14] have shown that the convergence of the series obtained depends on number-theoretic properties of $\theta$. If $a_{1}$ is a root of unity, even the formal calculation of the Schröder function is generally impossible and if $a_{1}=1$ this is always the case.

This paper is concerned entirely with the case $a_{1}=1$. The natural iterates $f_{n}(z)$ have been studied by Fatou [5] and others, but continuous iterates analytic at $z=0$ are not provided by the above Schröder method. There is, however, another approach based on the connexion between permutable functions and iterates (c.f. Hadamard [7], Szekeres [15]). We use the symbol $f \circ g$, where $f=\sum_{n=1}^{\infty} a_{n} z^{n}$ and $g=\sum_{n=1}^{\infty} b_{n} z^{n}$. are formal power series, to denote the formal power series $\sum_{n=1}^{\infty} c_{n} z^{n}$ obtained by substituting $g$ for $z$ in $f$ and rearranging according to powers of $z$. Since $c_{n}$ depends only on $a_{1}, \cdots a_{n}$ and $b_{1}, \cdots b_{n}$ there are no problems of convergence and the operation $f \circ g$ is associative. If $f$ is a given series with $a_{1}=1$ we may determine all series of the form $g$ which are permutable with $f$ in the sense

$$
f \circ g=g \circ f \text {. }
$$

This is done in $\$ 2$ and the set of such $g$ is found to include a family $F$ of series $f_{\lambda}$

$$
F: f_{\lambda}=z+\lambda a_{m+1} z^{m+1}+\sum_{n=m+2}^{\infty} b_{n}(\lambda) z^{n}, m \geqq 1, a_{m+1} \neq 0
$$

which satisfy

$$
f_{\lambda} \circ f_{\mu}=f_{\mu} \circ f_{\lambda}
$$

and may be regarded formally as a family of continuous interates. The discussion of the convergence of the series $f_{\lambda}$ in a given case is likely to be difficult since the rule of formation of the $b_{n}(\lambda)$ is complicated. In [1] the special case corresponding to $f_{1}=e^{x}-1$ was treated by indirect arguments to show that in that case only integral values of $\lambda$ yield convergent series (at least for real $\lambda$ ). This prompts the question: what $\lambda$-sets can arise as the sets of those values $\lambda$ for which the member $f_{\lambda}$ of a family $(F)$ has a positive radius of convergence? The answer is provided by $\S 6$, theorem 10: such a set consists of the whole $\lambda$-plane or of one or two dimensional lattice of points. In the first case $f(z)$ is embedded in a continuous group $F$ of iterates which are regular at $z=0$ and which possess a regular infinitesimal transformation $(\S 7)$. In the other cases no such embedding is possible.

Sections 3,4 contain extensions of the work of Fatou [5] which are used in the proof of theorem 10. Section 5 contains a proof that those $f_{\lambda}$ of a family which have positive radius of convergence satisfy certain conditions 
of boundedness (theorem 7) which are essential for the later proofs.

Besides studying iteration and permutability in the neighbourhood of a fixpoint one may consider e.g. permutable rational functions (Julia [10]), polynomials (Ritt [12] and Jacobsthal [9]) or entire functions (Baker [1] and Ganapathy Iyer [8]). In $\S 8$ some applications of our results to such global problems are considered.

\section{The formal power series}

The extent of the formal power series which are permutable with a given formal power series and the way in which these series form a continuous family are described in the following theorems.

THEOREM 1. If $f(z)$ is any formal series of the form

$$
f(z)=z+\sum_{n=m+1}^{\infty} a_{n} z^{n}, a_{m+1} \neq 0, m \geqq 1,
$$

then every formal series

$$
g(z)=\sum_{n=1}^{\infty} b_{n} z^{n}
$$

which is permutable with $f(z)$ in the sense of (4), has $b_{1}^{m}=1$.

PROoF: If $b_{k}, k \geqq 2$ is the first nonzero coefficient in (6), then the comparison of coefficients of powers of $z^{m+k}$ in (4) gives $k a_{m+1} b_{k}=0$, which is a contradiction. Thus only the case $k=1, b_{1} \neq 0$ is possible and the comparison of coefficients of $z^{m+1}$ in (4) gives $a_{m+1}\left(b_{1}-b_{1}^{m+1}\right)=0$, so that $b_{1}^{m}=1$.

THEOREM 2. Case $b_{1}=1$. With the notation of theorem 1: if $b_{1}=1$, then $b_{2}=\cdots=b_{m}=0$ while $b_{m+1}$ is arbitrary and to an arbitrary value $\lambda$ there is exactly one $g(z)$ with $b_{m+1}=\lambda a_{m+1}$. This $g(z)$ we denote by $f_{\lambda}(z)$ and it has the form

$$
f_{\lambda}(z)=z+\lambda a_{m+1} z^{m+1}+\sum_{n=m+2}^{\infty} b_{n}(\lambda) z^{n}
$$

where $b_{n}(\lambda)$ is a polynomial in $\lambda$ of degree at most $(n-m)$.

PROOF: Equating coefficients of $z^{m+2}$ in (4) gives $(m-1) a_{m+1} b_{2}=0$ and thus $b_{2}=0$ if $m>1$. Equating successively coefficients of $z^{m+j}$ for $j=3$, $\cdots, m$, gives $(m-j+1) a_{m+1} b_{j}=0$ and thus $b_{j}=0$. For $z^{2 m+1}$ the coefficients vanish identically, while for $k>m+1$ coefficients of $z^{m+k}$ give an equation of the form

$$
a_{m+1}(m+1-k) b_{k}=\sum_{j=m+1}^{k-1} c_{j} b_{j}+\sum d_{p a \ldots v} b_{1}^{p} b_{m+1}^{q} \cdots b_{k-1}^{p}
$$


where the non-negative integers $p, q, \cdots v$ satisfy

$$
m+1 \leqq p+q+\cdots+v \leqq m+k-1
$$

and

$$
p+(m+1) q+\cdots(k-1) v=m+k,
$$

while the $c_{j}$ and $d_{p e \ldots v}$ are dependent only on the coefficients $a_{n}$. It follows from (8) that $b_{m+1}$ is arbitrary and that once $b_{m+1}$ is given all other $b_{n}$, $n>m+1$ are determined inductively in a unique way. If we set $b_{m+1}=$ $\lambda a_{m+1}$ and calculate the $b_{n}$ they will be polynomials in $\lambda$ with coefficients depending only on the $a_{n}$. The degree of $b_{n}$ is at most $n-m$, as is proved by the following induction: for $n=m+1$ the degree is 1 . Suppose for $n<k$ the coefficient $b_{n}$ has degree at most $n-m$ in $\lambda$. In equation (8) every term of the first sum on the right has degree at most $(k-1)-m<k-m$ in $\lambda$ while the degree of any term of the second sum is (using (10))

$$
\begin{aligned}
D & \leqq q+2 r+\cdots+(k-1-m) v \\
& =p+(m+1) q+(m+2) r+\cdots+(k-1) v \\
& -p-m\{q+r+\cdots+v\} \\
& =m+k-p-m\{q+r+\cdots+v\} .
\end{aligned}
$$

If $q+r+\cdots+v=1$ then $p \geqq m$ from (9) so that $D \leqq k-m$, while if $q+r+\cdots+v \geqq 2$ then $D$ is at most $m+k-2 m=k-m$. Thus the right hand side of (8) has degree at most $(k-m)$ and the same is true of $b_{k}$.

We note some useful consequences of theorem 2 in

THEOREM 3. The series denoted in theorem 2 by $f_{\lambda}(z)$ coincide with the natural iterates in the case when $\lambda$ is a positive integer. For any two values $\lambda$ and $\mu$ one has

$$
f_{\lambda} \circ f_{\mu}=f_{\mu} \circ f_{\lambda}=f_{\mu+\lambda} \text {. }
$$

Proof: The natural iterate $f_{n}(z)$ defined by $f \circ f \circ \cdots \circ f$ with $n$ factors is a series of the form

$$
z+n a_{m+1} z^{m+1}+\cdots
$$

which is permutable with $f(z)$. By theorem 2 there is only one series with this property, namely the series $f_{\lambda}(z)$ with $\lambda=n$. The two are identical.

In the same way, since $f_{\lambda} \circ f_{\mu} \circ f=f_{\lambda} \circ f \circ f_{\mu}=f_{\lambda} \circ f_{\mu} \circ f$ and $f_{\mu} \circ f_{\lambda} \circ f=$ $f \circ f_{\mu} \circ f_{\lambda}$, while both $f_{\lambda} \circ f_{\mu}$ and $f_{\mu} \circ f_{\lambda}$ have the form

$$
z+(\lambda+\mu) a_{m+1} z^{m+1}+\cdots,
$$

equation (11) follows.

Combining equation (11) with theorem 2 one sees that the $f_{\lambda}(z)$ form a 
continuous group of formal power series, all of which are determined by any one member other than $f_{0}(z)$ which is the "identity" $z$. Thus each of the coefficients $b_{n}(\lambda)$ has the properties

$$
b_{n}(0)=0, b_{n}(1)=a_{n}, n \geqq m+1 .
$$

We now turn to the case where $b_{1}^{m}=1$ but $b_{1} \neq 1$. This arises only when $m>1$. We make a preliminary change of variables.

Lemma 1. There is a formal power series

$$
w=P(z)=z+\lambda_{2} z^{2}+\lambda_{3} z^{3}+\cdots
$$

such that if $f(z)$ is given by (5) with $m>1$, then $P \circ f \circ P_{-1}=F(w)$ has the form

$$
F(w)=w+A w^{m+1}+B w^{2 m+1}, A \neq 0 .
$$

PROOF: If $m>1$ we compare coefficients in $P \circ f=F \circ P$ and try to determine the $\lambda_{n}$ so as to give $F$ the form (14). Coefficients of $z^{k}$ for $k=1,2$, $\cdots m$ yield nothing, while $z^{m+1}$ yields $A=a_{m+1}$ and $z^{m+k}$ for $k=2, \cdots m$ yields an equation of the form

$$
(m+1-k) a_{m+1} \lambda_{k}=E\left(\lambda_{2}, \cdots \lambda_{k-1}, a_{m+1}, \cdots, a_{m+k}\right) .
$$

Thus $\lambda_{k}$ is determined for $k=2, \cdots m$. Equating coefficients of $z^{2 m+1}$ gives an equation which does not involve $\lambda_{m+1}$ but which determines $B$ in terms of the $a$ 's and $\lambda_{2}, \cdots, \lambda_{m} . \lambda_{m+1}$ may be given an arbitrary value. Equating coefficients of $z^{m+k}$ for $k=m+2, m+3, \cdots$ yields an equation

$$
(m+1-k) a_{m+1} \lambda_{k}=E\left(\lambda_{2}, \ldots, \lambda_{k-1}, B, a_{m+1}, \ldots, a_{m+k}\right),
$$

which determines all further $\lambda_{k}$.

TheOREM 4. For $f(z)$ as in (5) theorem 1 , there is to each value of $b_{1}$ which satisfies $b_{1}^{m}=1$ exactly one formal power series

$$
H(z)=\sum_{n=1}^{\infty} b_{n} z^{n}
$$

for which $H_{m}(z) \equiv z$ while $H \circ f=f \circ H$. The series $g(z)$ of the form (6) which are permutable with $f(z)$ are exactly the series $H \circ f_{\lambda}$, where $f_{\lambda}$ has the meaning described in theorem 2.

Proof: Let $b_{1}$ be any root of $b_{1}^{m}=1$ and let $P(z)$ be any power series (13) satisfying the conditions of Lemma 1. If $F=P \circ f \circ P_{-1}$ and $g(z)=b_{1} z+\ldots$ is permutable with $f(z)$, then $G=P \circ g \circ P_{-1}=b_{1} z+\ldots$ is permutable with $F$. Since $F(z)$ has the form (14) it is permutable with the series $b_{1} z$ and its $(m-1)$-th iterate $b_{1}^{-1} z$, and hence with $\left(b_{1}^{-1} z\right) \circ G=b_{1}^{-1} G$. 
Since $b_{1}^{-1} P \circ g \circ P_{-1}$ commutes with $F$, it follows that $k=P_{-1} \circ b_{1}^{-1} P \circ g$ $\circ P_{-1} \circ P=P_{-1} \circ b_{1}^{-1} P \circ g$ commutes with $f$. Now $k=z+\ldots$ and by theorem 2 must be identical with one of the $f_{\lambda}$. Hence we have $g=P_{-1} \circ b_{1} P \circ f_{\lambda}=H \circ f_{\lambda}$ where $H \equiv P_{-1} \circ b_{1} P$. Clearly $H_{m}=P_{-1} \circ b_{1}^{m} P \equiv z$. Also $H \circ f=P_{-1} \circ b_{1} P \circ f \circ P_{-1} \circ P=P_{-1} \circ b_{1} F \circ P=P_{-1} \circ F \circ\left(b_{1} z\right) \circ P=P_{-1}$ $\circ F \circ P_{-1} \circ b_{1} P=f \circ H$. It remains to prove the uniqueness of $H=b_{1} z+\ldots$ Suppose $K=b_{1} z+\ldots$ also commutes with $f$ and satisfies $K_{m} \equiv z$. Then $H \circ K_{-1}$ commutes with $f$ and has an expansion $z+\ldots$, so that it must be identical with some $f_{\lambda}$. We shall prove in theorem 5 that $H$ and $K$ commute so that

$$
\left(H \circ K_{-1}\right)_{m}=f_{m \lambda}=\left(H_{m} \circ K_{-m}\right) \equiv z \text { and } \lambda=0 .
$$

THEOREM 5. Any two series $g(z)$ and $h(z)$, each of the form (6) and permutable with $f(z)$ of the form (5), satisfy $g \circ h=h \circ g$.

PROof: If $g$ and $h$ both have the form $f_{\lambda}$ the result follows from theorem 3. If $h=H(z)$ is of the form (15) and if $g=f_{\lambda}$ then by the uniqueness result of theorem 2 there is a $\mu$ such that

$$
H \circ f_{\lambda} \circ H_{-1}=f_{\mu} \text {. }
$$

If $H$ and $H_{-1}$ have expansions

$$
\begin{gathered}
H=\sum_{n=1}^{\infty} b_{n} z^{n}, \\
H_{-1}=b_{1}^{-1} z+\sum_{n=2}^{\infty} \beta_{n} z^{n},
\end{gathered}
$$

then the comparison of terms in $z^{m+1}$ in (16), using (7) and the fact that $H$ and $H_{-1}$ are formal inverses, gives

$$
\lambda a_{m+1}=\mu a_{m+1}
$$

so that $H \circ f_{\lambda}=f_{\lambda} \circ H$.

Further, if $H, K$ are two solutions of $H_{m}=K_{m}=z$ which are permutable with $f$, then $H \circ K=b z+\cdots$ where $b^{m}=1$. Thus $(H \circ K)_{m}=z+$ higher terms and is permutable with $f$ so that from theorem 2 there is a $\lambda$ such that $(H \circ K)_{m}=f_{\lambda}$. But then $(K \circ H)_{m}=K \circ(H \circ K)_{m} \circ K_{-1}=K \circ f_{\lambda} \circ K_{-1}$ $=f_{\lambda}$ since by the preceding part of the proof $K$ is permutable with $f_{\lambda}$. Now because its expansion has 1 for the coefficient of $z, H \circ K \circ H_{-1} \circ K_{-1}$ must be $f_{\alpha}$ for some $\alpha$. Then $(H \circ K)_{m}=\left(f_{\alpha} \circ K \circ H\right)_{m}=f_{m \alpha} \circ(K \circ H)_{m}$ since $f_{\alpha}$ is permutable with both $H$ and $K$. This means that $\lambda=m \alpha+\lambda$ so that $\alpha=0$, $f_{\alpha} \equiv z$ and $H \circ K=K \circ H$. This is the result which was needed in the last step of theorem 4, which is now completely proved. Using the result of theorem 4 it now follows that in every case the statement of theorem 5 holds. 
It may be noted that the result of theorem 5 depends essentially on the restriction to series having a common fixpoint (namely $z=0$ ). It is not always true, for example, that if the entire functions $g(z), h(z)$ are permutable with the entire function $f(z)$, then $g(z)$ is permutable with $h(z)$. A counter example is provided by the functions

$$
\begin{aligned}
& f(z)=z+\sin z+\sin (z+\sin z), \\
& g(z)=-z-\sin z, \\
& h(z)=2 \pi-z-\sin z,
\end{aligned}
$$

for which $g_{2}(z)=h_{2}(z)=f(z)$. In fact $-g(z)$ and $h(z)$ have no common fixpoint and $g(h)-h(g) \equiv-4 \pi$. The purely formal considerations of this section therefore have consequences for such global problems as the set of entire functions which commute with a given entire function $F(z)$. As an example we prove:

THEOREM 6. If $F(z)$ is an entire function exactly one of whose fixpoints has multiplier 1 , then any two non-constant entire functions permutable with $F(z)$ are permutable with one another.

PRoof: We may suppose the fixpoint in question to be $z=0$ for if $F(z)$ has fixpoint $c$ with $F^{\prime}(c)=1$, then $f(z)=F(z+c)-c$ has fixpoint $z=0$ with multiplier $f^{\prime}(0)=1$. Also if $F(G(z))=G(F(z))$ and if $g(z)=G(z+c)-c$, then $f(g(z))=g(f(z))$ and conversely.

If $G(z)$ is any non-constant entire function satisfying $F(G(z))=G(F(z))$, it follows from $F(0)=0$ that $G(0)$ is a fixpoint of $F(z)$. If one differentiates the identity $F(G(z))=G(F(z))$ and sets $z=0$ one obtains $F^{\prime}(G(0)) G^{\prime}(0)=$ $G^{\prime}(0) F^{\prime}(0)=G^{\prime}(0)$, so that $F^{\prime}(G(0))=1$ unless $G^{\prime}(0)=0$. Since $G(z)$ is not a constant there is a least value of $m$ such that the derivative $G^{(m)}(0) \neq 0$ and $m$-fold differentiation of the above identity yields for $z=0$ the equation

$$
F^{\prime}(G(0)) G^{(m)}(0)=G^{(m)}(0),
$$

so that $F^{\prime}(G(0))=1$ and $F(G(0))=G(0)$. By hypothesis the only fixpoint of multiplier 1 of $F(z)$ is $z=0$, and hence $G(0)=0$. The expansion of $G(z)$ in powers of $z$ has no constant term and is therefore contained among the formal series of the form (6) satisfying $G \circ F=F \circ G$. Since any two of these series are formally permutable, any two representing entire functions will be permutable as entire functions.

We shall return to such global applications after considering the convergence of the formal series obtained in theorems 1 to 5 .

\section{Natural iteration near a fixpoint of multiplier 1}

The results of this section are mainly due to Fatou [5] but are developed in forms suitable to the applications made of them in $\S 4$. It is convenient 
to put the fixpoint at infinity and to begin by treating a function defined for all sufficiently large values of $z$ by the convergent series

$$
F(z)=z+a+\frac{b}{z}+\frac{c}{z^{\alpha}}+\cdots, \alpha>1, a>0 .
$$

The expansion in descending powers of $z$ may contain fractional powers which can be assumed to be made single valued by cutting the region of convergence along the negative real axis. In fact we assume all the exponents of $z$ in (17) to be multiples of $1 / n$ for some integer $n>1$. Now to a given value $\theta$ with $0<\theta<\pi / 2$ there is a positive constant $K$ such that for $|z| \geqq K$ the series (17) converges and

$$
|F(z)-z-a|<a \sin \theta \text { for }|z| \geqq K .
$$

We denote by $\mathbb{C}$ the curve formed by the circular arc

$$
K e^{i t},|t| \leqq \frac{\pi}{2}-\theta,
$$

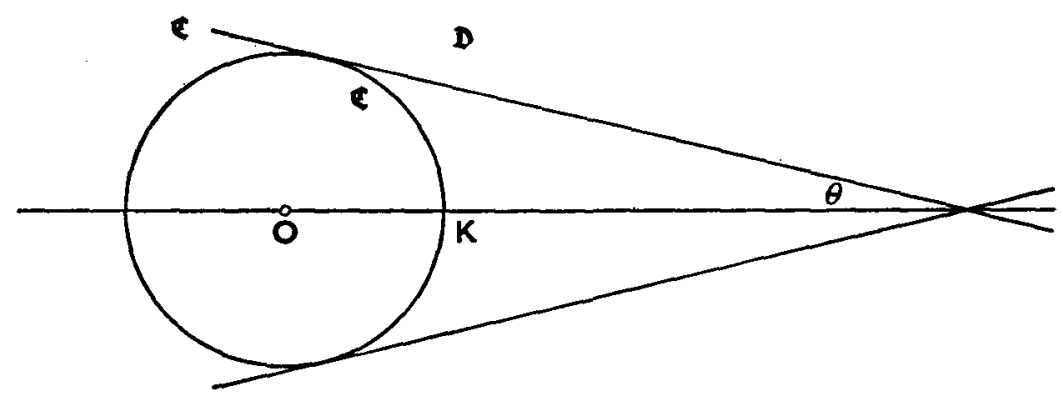

Fig. 1.

together with the two infinite segments of the tangents at the extremities of this arc which extend from the extremities to infinity in the left half plane. The region $\mathscr{D}=\mathscr{D}(\theta, K)$ is defined to be the region bounded on the left by $\mathfrak{C}$.

LEMMA 2. If $F(z)$ has the form (17), $\theta$ satisfies $0<\theta<\pi / 2$ and $\mathfrak{D}=$ $D(\theta, K)$ is defined as above, then for all sufficiently large $K$

$$
F_{n}(z) \in \mathfrak{D}, n=1,2, \cdots
$$

and

$$
\operatorname{Re} F_{n}(z) \rightarrow \infty \quad \text { as } n \rightarrow \infty
$$

for all $z$ in the closure $\bar{D}$ of $\mathfrak{D}$.

PROOF: From (18) follows (19) in the case $n=1$ and by inductive iteration of this result the other cases also follow. From (18): 


$$
\operatorname{Re}\{F(z)-z\}>a(1-\sin \theta) \text { for } z \in \bar{D}
$$

and since $F_{m}(z) \in \mathcal{D}$ for $m=1,2, \cdots$ one has

$$
\operatorname{Re}\left\{F_{m+1}(z)-F_{m}(z)\right\}>a(1-\sin \theta) \text { for } z \in \bar{D}, m=0,1, \cdots, n-1 \text {. }
$$

Adding these inequalities one obtains

$$
\operatorname{Re}\left\{F_{n}(z)-z\right\}>n(1-\sin \theta) \text { for } z \in \overline{\mathfrak{D}}, n=1,2, \cdots
$$

from which (20) follows.

By a straightforward calculation Fatou [5] shows that if $\mathfrak{D}=\mathfrak{D}(\theta, K)$ is a region satisfying lemma 2 , then the limit

$$
A(z)=\lim _{n \rightarrow \infty}\left\{F_{n}(z)-n a-\frac{b}{a} \log n\right\}
$$

exists uniformly for $z \in \mathscr{D}$. The function $A(z)$ is regular in $\mathfrak{D}$. If $K$ is sufficiently large $F(z)$ is schlicht in $D$ and so consequently are all $F_{n}(z), n=1$, $2, \cdots$ It follows that $A(z)$ is either schlicht in $D$ or a constant, but from (22) and (17) one sees that $A(z)$ satisfies

$$
A(F(z))=A(z)+a, z \in \mathfrak{D}
$$

and is not a constant but a schlicht function.

Lemma 3. If $F(z)$ has the form (17) and $\theta$ satisfies $0<\theta<\pi / 2$, then for all sufficiently large $K$ the domain $D=D(\theta, K)$ satisfying lemma 2 has the further property that the function $A(z)$ satisfying (22) and (23) is schlicht in $D$ and $A^{\prime}(z) \rightarrow 1$ uniformly as $z \rightarrow \infty$ in $D$.

Proof: We have only to prove that $A^{\prime}(z) \rightarrow 1$ as $z \rightarrow \infty$ in $\mathfrak{D}$. One may assume $K$ to be so large that for all $z \in \mathscr{D}$

$$
1-\frac{B}{|z|^{2}}<\left|F^{\prime}(z)\right|<1+\frac{B}{|z|^{2}},
$$

where $B>0$ is a suitable contant; and $K$ can be enlarged further if neces. sary so that $B<K^{2}$. Then

$$
\frac{B}{|z|^{2}}<1 \text { for } z \in \mathfrak{D} \text {. }
$$

Now

$$
F_{n}^{\prime}(z)=\prod_{j=0}^{n-1} F^{\prime}\left(F_{j}(z)\right)
$$

and if $z=z_{0} \in \mathfrak{D}$, then for $j=1,2, \cdots$ we have $z_{j}=F_{f}(z) \in \mathfrak{D}$ and 


$$
\prod_{j=0}^{n-1}\left(1-B\left|z_{j}\right|^{-2}\right)<\left|F_{n}^{\prime}(z)\right|<\prod_{j=0}^{n-1}\left(1+B\left|z_{j}\right|^{-2}\right) .
$$

But from (22) $A^{\prime}(z)=\lim _{n \rightarrow \infty} F_{n}^{\prime}(z)$ and hence

$$
\prod_{j=0}^{\infty}\left(1-B\left|z_{j}\right|^{-2}\right) \leqq\left|A^{\prime}(z)\right| \leqq \prod_{j=0}^{\infty}\left(1+B\left|z_{j}\right|^{-2}\right) .
$$

Enlarging $K$ still further will not alter the validity of the above arguments for $z \in \mathfrak{D}(\theta, K)=\mathscr{D}$ and we suppose $K$ so large that for $|z|>K$ and in particular for $z \in \mathscr{D}$ one has

$$
|F(z)-z-a|<a \sin \theta / 2 .
$$

For any $z_{0} \in \mathscr{D}$ the points $z_{n}=F_{n}\left(z_{0}\right)$ lie in an angle

$$
\left|\arg \left(z-z_{0}\right)\right|<\theta / 2
$$

whose vertex is at $z_{0}$ and whose bisector extends in the direction of the positive real axis. Thus if

$$
\left|\arg z_{0}\right| \leqq(\pi-\theta) / 2, \quad z_{0} \in \mathfrak{D},
$$

then

$$
\left|z_{0}\right|<\left|z_{1}\right|<\cdots<\left|z_{n}\right|<\left|z_{n+1}\right|<\cdots
$$

If on the other hand

$$
\left|\arg z_{0}\right|>(\pi-\theta) / 2, z_{0} \in \mathfrak{D},
$$

then the radius vector from 0 to $z_{0}$ makes with the nearer arm of the angle (29) an angle $\gamma$ lying between $\theta / 2$ and $\pi / 2$. Since all $z_{n}$ lie in the angle (29) and the nearest point of (29) to 0 has modulus $\left|z_{0}\right| \sin \gamma$ it follows that in this case (32) one has

$$
\left|z_{n}\right| \geqq\left|z_{0}\right| \sin \gamma>\left|z_{0}\right| \sin \theta / 2
$$

Thus for any $z_{0} \in \mathscr{D}$ we have either (30) or (32) and hence either (31) or (33) so that in any case

$$
\left|z_{n}\right|>\left|z_{0}\right| \sin \theta / 2=\left|z_{0}\right| t \text { for } n=1,2, \cdots
$$

where $t=\sin \theta / 2$. From (21) with $s=1-\sin \theta$ one has

$$
\left|F_{n}\left(z_{0}\right)\right| \geqq \operatorname{Re} F_{n}\left(z_{0}\right)>n s-\left|z_{0}\right| \text { for } n>\left|z_{0}\right| / s .
$$

For $n>\left[2\left|z_{0}\right| / s\right]+1=N$, where the square bracket means "integral part of $2\left|z_{0}\right| / s^{\prime \prime}$, one has $\left|z_{0}\right|<s n / 2$ and from (35) follows

$$
\left|F_{n}\left(z_{0}\right)\right|>n s / 2 \text { for } n>N \text { and } z_{0} \in \mathfrak{D} \text {, }
$$

Using (34) and (36) for $z_{0} \in \mathfrak{D}$ one has 


$$
\begin{aligned}
Q=\sum_{n=0}^{\infty}\left|z_{n}\right|^{-2} & =\sum_{0}^{N-1}+\sum_{N}^{\infty} \\
& <\frac{N}{t^{2}\left|z_{0}\right|^{2}}+\sum_{N}^{\infty} \frac{4}{(n s)^{2}} \\
& <\frac{N}{t^{2}\left|z_{0}\right|^{2}}+\frac{4}{s^{2}(N-1)} \\
& <\frac{2}{s t^{2}\left|z_{0}\right|}+\frac{1}{t^{2}\left|z_{0}\right|^{2}}+\frac{4}{\left(2\left|z_{0}\right|-s\right) s}
\end{aligned}
$$

Thus the series $Q$ converges for $x_{0} \in \mathscr{D}$ and $Q \rightarrow 0$ uniformly as $z \rightarrow \infty$ in $\mathscr{D}$ so that the infinite products in (27) converge. Since the left hand product of (27) is greater than $1-B Q$ while the right hand one is less than $\exp (B Q)$ the lemma is established.

\section{Transformation of the permutable series}

A change of variables is now made to replace the series of a family (7) by series (46) of the form (17). In the commuting family

$$
f_{\lambda}(z)=z=+\lambda a_{m+1} z^{m+1}+\sum_{n=m+2}^{\infty} b_{n}(\lambda) z^{n}
$$

put

$$
\begin{gathered}
w=P(z)=z+\sum_{n=2}^{m} A_{n} z^{n}, \\
z=P_{-1}(w)=w+\sum_{n=2}^{\infty} A_{n}^{\prime} z^{n},
\end{gathered}
$$

where the $A_{n}$ are so chosen that

$$
P \circ f_{1} \circ P_{-1}(w)=Q_{1}(w)
$$

has the form

$$
Q_{1}(w)=w+a_{m+1} w^{m+1}+\sum_{n=2 m+1}^{\infty} B_{n} w^{n} .
$$

The possibility of so doing has been shown by Fatou $[5, \S \S 11,12]$. For $m=1$ the transformation reduces to $w=z$. If for each value of $\lambda$ one sets

$$
Q_{\lambda}(w)=P \circ f_{\lambda} \circ P_{-1}(w),
$$

then the $Q_{\lambda}$ form a family of mutually permutable series whose expansions begin

$$
Q_{\lambda}(w)=w+\lambda a_{m+1} w^{m+1}+\cdots
$$


By direct calculation in $Q_{1} \circ Q_{\lambda}=Q_{\lambda} \circ Q_{1}$ one finds that all $Q_{\lambda}$ have the form

$$
Q_{\lambda}(w)=w+\lambda a_{m+1} w^{m+1}+\sum_{n=2 m+1}^{\infty} B_{n}(\lambda) w^{n} .
$$

By moving the fixpoint $w=0$ to infinity and rotating axes:

$$
w=e^{-i \gamma} v^{-1}, R_{\lambda}(v)=e^{-i \gamma} Q_{\lambda}^{-1}\left(e^{-i \gamma} v^{-1}\right), \gamma \text { constant, }
$$

where $Q_{\lambda}^{-1}$ is the reciprocal of $Q_{\lambda}$, we obtain

$$
R_{\lambda}(v)=v-\lambda a_{m+1} \exp (-i \gamma m) v^{1-m}+\sum_{n=2 m}^{\infty} c_{n}(\lambda) v^{1-n} .
$$

We choose the constant $\gamma$ in such a way that

$$
a=-a_{m+1} \exp (-i \gamma m)>0
$$

and (42) becomes

$$
R_{\lambda}(v)=v+\lambda a v^{1-m}+\sum_{n=2 m}^{\infty} c_{n}(\lambda) v^{1-n}
$$

Finally we set

$$
v=t^{1 / m}, F_{\lambda}(t)=\left\{R_{\lambda}\left(t^{1 / m}\right)\right\}^{m},
$$

and obtain an expression

$$
F_{\lambda}(t)=t+\lambda a m+\sum_{n=0}^{\infty} D_{n}(\lambda) t^{-(m+n) / m},
$$

in which the $D_{n}(\lambda)$ are uniquely determined by the formal transformations $(37)-(45)$. Moreover, if for a value $\lambda$ the series $f_{\lambda}$ of (7) has a positive radius of convergence then there is a $K>0$ such that for any choice of $t^{1 / m}$ in (46) the series $F_{\lambda}$ converges for $|t| \geqq K$, and conversely. We introduce the notation: $\mathfrak{\Re}=\mathfrak{\Re}\left(f_{\lambda}\right)$ is the set of values $\lambda$ for which the series $f_{\lambda}$ of the family (7) or equivalently $F_{\lambda}$ of (46) have positive radius of convergence.

Further, the notation

$$
T_{j}=t_{j}^{1 / m}=t^{1 / m}, \quad j=1,2, \cdots, m
$$

is introduced for that branch of the function $t^{1 / m}$ for which

$$
(2 j-3) \pi / m<\arg T_{j} \leqq(2 j-1) \pi / m .
$$

For $\lambda \epsilon \Re, 1 \leqq j \leqq m$ the substitution of (47) in (46) gives a function

$$
F_{\lambda}^{\prime}(t)=t+\lambda a m+\sum_{n=0}^{\infty} D_{n} T_{j}^{-(m+n)}
$$


regular and single valued in neighbourhood of $t=\infty$ cut along the negative real axis.

For $0<\theta<\pi / 2$ and all sufficiently large $K$ the domain $\mathscr{D}(\theta, K)$ in the $t$-plane is mapped by the composition of the transformations (37), (41), $(45)-(47)$ one to one and conformally on to a domain $\mathfrak{B}(j, \theta, K)$ of the $z$ plane bounded by a simple closed curve analytic except at $z=0$ where two boundary arcs meet in the directions

$$
\left\{\begin{array}{l}
\arg z=-\gamma+\theta / m-(2 j-1) \pi / m \\
\arg z=-\gamma-\theta / m-(2 j-3) \pi / m
\end{array}\right.
$$

For $K>L$ one has $\mathfrak{D}(\theta, K) \subset \mathfrak{D}(\theta, L)$ and hence

$$
\mathscr{B S}(j, \theta, K) \subset \mathscr{B}(j, \theta, L) \text {. }
$$

By taking $K$ sufficiently large one may make $\mathbb{S}(j, \theta, K)$ interior to any given circle $|z|\langle\rho, \rho\rangle 0$. For large $K$ the $m$ domains $\&(j, \theta, K)$ exist and are ranged about $z=0$ in a star $\mathfrak{S}(\theta, K)$ :

$$
\mathfrak{S}(\theta, K)=\bigcup_{j=1}^{m} \mathfrak{S}(j, \theta, K) .
$$

Figure 2 illustrates $S(\theta, K)$ in the case $m=\mathbf{3}, \gamma=\mathbf{0}$

LEMMA 4. If $f_{\lambda}$ and $F_{\lambda}^{j}, 1 \leqq j \leqq m$, are members of the families (7) and (49) respectively, if $\lambda \in \Re$ and $0<\theta<\pi / 2$, then for all sufficiently large $K$ one has

$$
F_{\lambda}^{j}\{\mathfrak{D}(\theta, K+2|\lambda| a m)\} \subset \mathcal{D}(\theta, K),
$$

while if $\lambda>0$

$$
F_{\lambda}^{j}\{\mathfrak{D}(\theta, K)\} \subset \mathfrak{D}(\theta, K) .
$$

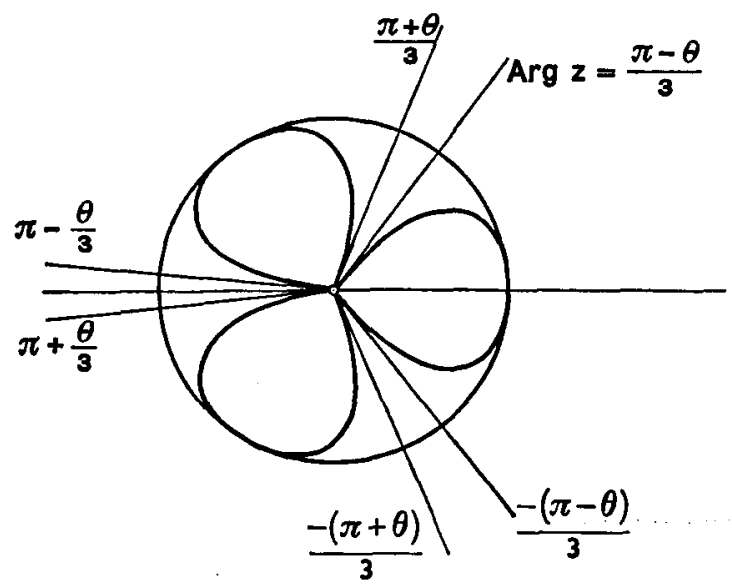

Fig. 2. 
Further the functions $f_{\lambda}(z)$ and $F_{\lambda}^{j}(t)$ represent the same transformation in $z$ and $t$ coordinates respectively so that

$$
f_{\lambda}\{\leftrightarrow(j, \theta, K+2|\lambda| a m)\} \subset \circlearrowleft(j, \theta, K)
$$

and

$$
f_{\lambda}\{\subseteq(\theta, K+2|\lambda| a m)\} \subset \subseteq(\theta, K)
$$

hold for all sufficiently large $K$, while if $\lambda>0$ :

$$
f_{\lambda}\{\varsigma(\theta, K)\} \subset \Im(\theta, K) \text {. }
$$

Proof: Equations (52) and (43) follow from the form of (49) and the fact that $a>0$. We now show the equivalence of the functions $F_{\lambda}^{j}(t)$ and $f_{\lambda}(z)$ for $\lambda \in \Re$. The functions $T_{j}(t)=t^{1 / m}$ of $(47)$ and $R_{\lambda}(v)=R_{\lambda}\left(T_{1}(t)\right)$ of (44) are regular for $t \in \mathscr{D}(\theta, K)=\mathscr{D}$ if $K$ is large enough. Also $t_{1}=F_{\lambda}^{j}(t)$ in (49) is regular for $t$ in $\mathfrak{D}=\mathscr{D}_{\lambda}^{j}(\theta, K) \supset \mathfrak{D}_{\lambda}^{j}(\theta, K+2|\lambda| a m)=\mathscr{D}_{0}$, and for $t \in \mathfrak{D}_{0}$ we have $t_{1} \in \mathfrak{D}$ by (52). We also suppose $K$ to be so large that the series of transformations (37), (41), (45), (47) map $\mathfrak{D}$ one to one and conformally on to $\mathbb{S}(j, \theta, K)$ as explained immediately after equation (49). If the successive images of $t$ and $t_{1}$ under these transformations are $v$ and $v_{1}, w$ and $w_{1}$, $z$ and $z_{1}$ respectively, we have to show that for $t \in \mathscr{D}_{0}$ and $t_{1}=F_{\lambda}^{j}(t)$ one has

$$
z_{1}=f_{\lambda}(z)
$$

This done, (54) and (55) follow at once from (52). Now

$$
v_{1}=\left(t_{1}\right)_{j}^{1 / m}=T_{j}\left(t_{1}\right)=T_{j}\left(F_{\lambda}^{j}(t)\right)
$$

is regular for $t \in \mathscr{D}_{0}$ since $t_{1} \in \mathfrak{D}$ and $\mathfrak{D}$ does not contain the origin. But from (45) follows

$$
v_{1}^{m}=t_{1}=\left\{R_{\lambda}\left(T_{\jmath}(t)\right)\right\}^{m}
$$

whence

$$
v_{1}=\eta R_{\lambda}(v)=\eta R_{\lambda}\left(T_{j}(t)\right)
$$

where $\eta^{m}=1$. Since both sides of (56) are regular for $t \in \mathscr{D}_{0}$ the same value of $\eta$ must be valid for all $t \in D_{0}$. But as $t \rightarrow \infty$ along the positive real axis it follows from (49) and (47) that

while from (44)

$$
\arg v_{1}=\arg T_{1}\left(t_{1}\right) \sim \arg T(t),
$$

$$
\arg R_{\lambda}(v)=\arg R_{\lambda}\left(T_{j}(t)\right) \sim \arg T(t) .
$$

Hence $\eta=1$. Thus $v_{1}=R_{\lambda}(v)$ and since the remaining transformations (37) and (41) are one to one in the regions under discussion, it follows that $z_{1}=f_{\lambda}(z)$. 
A consequence of Lemma 4 is that $F_{n \lambda}^{\prime}(t)$ is indeed the $n$-th iterate of $F_{\lambda}^{j}(t)$ and $F_{\lambda}^{j}(t)$ commutes with $F_{\mu}^{j}(t)$ in suitable regions of the form $\mathscr{D}(\theta, K)$. We note the following

LEMma 5. With the assumptions of lemma 4 , if $\lambda \in \Re$ and $1 \in \Re$, then for all sufficiently large $K$ one has for all $t \in \mathfrak{D}(\theta, K+2|\lambda| a m)$

$$
F_{n}^{j}\left(F_{\lambda}^{j}(t)\right)=F_{\lambda}^{j}\left(F_{n}^{j}(t)\right) \in \mathfrak{D}(\theta, K), n=0,1,2, \cdots
$$

Proof: For $n=0$ the result is included in lemma 4, since $F_{0}^{j}(t)=t$. We suppose the result holds for $0,1, \cdots, n-1$. We also suppose $K$ so large that for all $L>K$ equation (53) holds with $\lambda=1$ :

$$
F_{1}^{\mathfrak{y}}(\mathfrak{D}(\theta, L)) \in \mathfrak{D}(\theta, L) .
$$

Then for $t \in \mathfrak{D}(\theta, K+2|\lambda| a m), F(t) \in \mathscr{D}(\theta, K+2|\lambda| a m)$ and by the induction hypothesis

$$
F_{\lambda}^{j}\left(F_{n-1}^{j}\left(F_{1}^{j}(t)\right)\right)=F_{n-1}^{j}\left(F_{\lambda}^{j}(F(t))\right)=F_{n-1}^{j}\left(F^{j}\left(F_{\lambda}^{j}(T)\right)\right)=F_{n}^{j}\left(F_{\lambda}^{j}(t)\right)
$$

\section{Normality of the family $f_{\lambda}$}

\section{THEOREM 7. Let}

$$
f_{\lambda}(z)=z+\lambda a_{m+1} z^{m+1}+\sum_{n=m+1}^{\infty} a_{n} z^{n}, a_{m+1} \neq 0, m \geqq 1,
$$

be a commuting family of formal power series as in theorems 1 and 2. For $p>0$ let $\Re_{p}$ be the class of complex $\lambda$ with $|\lambda| \leqq p$ for which $f_{\lambda}(z)$ has positive radius of convergence. Then there exist constants $\rho>0$ and $M>0$ such that

$$
f_{\lambda}(z) \text { converges in }|z| \leqq \rho \text { for all } \lambda \in \mathfrak{\Re}_{p}
$$

and

$$
\left|f_{\lambda}(z)\right|<M \text { uniformly for all }|z| \leqq \rho \text { and all } \lambda \in \Re_{p} \text {. }
$$

Proof: If $\Re_{p}$ contains only $\lambda=0$ there is nothing to prove. In the other cases there is no loss in generality if we assume $\lambda=1$ to be contained in $\Omega_{p}$ for there is certainly a $\mu \neq 0, \mu \in \Re_{p}$, and the substitutions $\lambda=\mu \lambda^{\prime}, f_{\mu}(z)=$ $g_{1}(z), f_{\lambda}(z)=g_{\lambda}(z)$ reduce the general case to the special one (with a different value of $p$ ).

We assume then that $p>1$ and that $\lambda=1$ is contained in $\Re_{p}$. We choose a fixed integer $j$ satisfying $1 \leqq j \leqq m$ and set

$$
F(t)=F_{1}^{j}(t)=t+m a+\sum_{n=0}^{\infty} D_{n}(1) T_{j}(t)^{-m+n}
$$

for that function (49) for which $\lambda=1 . F(t)$ is simply the function $f_{1}(z)$ 
represented in a different system of variables. Since $m a>0$ by (43), the series (58) is of the form (17) and lemma 3 applies. Thus if $\theta$ is a fixed number satisfying $0<\theta<\pi / 2$, the domain $\mathscr{D}=\mathscr{D}(\theta, K)$ described in $\S 3$ will for all sufficiently large $K$ be a domain of existence and univalence of $F(t)$ and of the function $A(t)$ (defined by (22) with $z$ replaced by $t$ ). Since $A^{\prime}(t) \rightarrow 1$ as $t \rightarrow \infty$ in $\mathfrak{D}$ one may take $K$ so large that for all $t \in \bar{D}$ one has

$$
\left|\arg A^{\prime}(t)\right|<\theta / 2,\left|A^{\prime}(t)\right|>\frac{1}{2}>0 .
$$

These conditions ensure that $s=A(t)$ maps $\mathscr{D}$ one to one and conformalyl on to a region $B$ of the $s$-plane with the property: $\mathfrak{B}$ is the region lying to the right of a simple analytic curve $\mathfrak{L}$ which is cut just once by every parallel to the real axis and whose limiting slope at infinity is $-\tan \theta$ in the upper half-plane and $+\tan \theta$ in the lower half-plane (c.f. Fig. 3). By (59) the

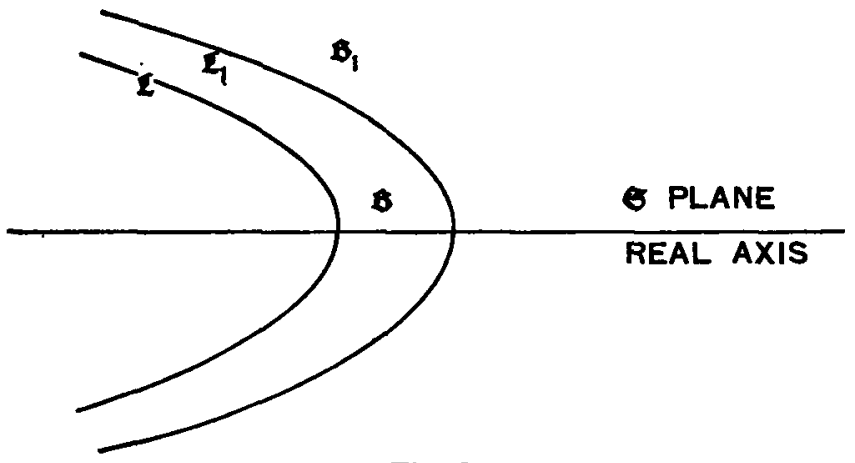

Fig. 3.

slope of $\mathfrak{L}$ has a numerical value greater than $\theta / 2$.

We denote by $\mathfrak{B}_{1}$ the region formed by displacing $\mathfrak{B}$ an amount $p \overrightarrow{a m}$, where $\bar{a}=a /(\sin \theta / 2)$, in the direction of the positive real axis. The distance of a point of $\mathfrak{B}_{1}$ from $\mathfrak{Q}$ is at least pam.

We now suppose $\lambda$ to be any fixed value in $\Re_{p}$. By lemma 5 there is an $L$ greater than the $K$ used above in the definition of $\mathfrak{D}(\theta, K)$ and $\mathfrak{B}$, such that for $t \in \mathscr{D}(\theta, L+2|\lambda| a m)$ one has

$$
F_{n}\left(F_{\lambda}^{j}(t)\right)=F_{\lambda}^{j}\left(F_{n}(t)\right) \in \mathfrak{D}(\theta, L) \subset \mathfrak{D}(\theta, K), n=0,1,2, \cdots
$$

Now by (22).

$$
A(t)=\lim _{n \rightarrow \infty}\left\{F_{n}(t)-n a m-\frac{D_{0}(1)}{a} \log n\right\}, \quad t \in \mathfrak{D}(\theta, K) .
$$

For $t \in \mathfrak{D}(\theta, L+2|\lambda|$ am $)$ one has $F_{\lambda}^{j}(t)$ in $\mathfrak{D}(\theta, K)$ by $(60)$ and from $(60)$, $\left(22^{\prime}\right)$ one has 


$$
\begin{aligned}
A\left(F_{\lambda}^{j}(t)\right) & =\lim _{n \rightarrow \infty}\left\{F_{n}\left(F_{\lambda}^{j}(t)\right)-n a m-\frac{D_{0}(1)}{a} \log n\right\} \\
& =\lim _{n \rightarrow \infty}\left\{F_{\lambda}^{j}\left(F_{n}(t)\right)-n a m-\frac{D_{0}(1)}{a} \log n_{n}\right\},
\end{aligned}
$$

whence by (49)

$$
A\left(F_{\lambda}^{j}(t)\right)=A(t)+\lambda a m .
$$

Since the functions in (61) are regular the equation not only holds in $\mathscr{D}(\theta, L+2|\lambda| a m)$ but can be used in the form

$$
F_{\lambda}^{j}(t)=A_{-1}\{A(t)+\lambda a m\}
$$

to give a regular continuation of $F_{\lambda}^{\prime}(t)$ throughout the simply connected domain $A_{-1}\left(\mathscr{B}_{1}\right)$ of the $t$-plane.

We show that $A_{-1}\left(\mathfrak{B}_{1}\right) \supset \mathcal{D}(\theta, K+2 p \bar{a} m)$. Let $t_{1}$ be a finite point on the boundary of $A_{-1}\left(\mathfrak{B}_{1}\right)$ and let $s_{1}=A\left(t_{1}\right)$ so that $s_{1}$ is on the boundary of $\mathfrak{B}_{1}$. Put $s_{2}=s_{1}-p \bar{a} m$ (on the boundary of $\mathfrak{B}$ ) and $t_{2}=A_{-1}\left(s_{2}\right)$. The segment $\Gamma: s_{1} s_{2}$ of length päm joins the boundaries of $\mathscr{B}$ and $\mathscr{B}_{1}$ and its image $A_{-1}(\Gamma)$ joins the boundaries of $\mathfrak{D}(\theta, K)=A_{-1}(\mathfrak{B})$ and $A_{-1}\left(\mathfrak{B}_{1}\right)$. From (59) the length of $A_{-1}(\Gamma)$ is less than twice the length of $\Gamma$ and so is less than 2 päm. Our assertion follows.

Certainly then $F_{\lambda}^{\prime}(t)$ is regular in $\mathscr{D}(\theta, K+2 p \bar{a} m)$ and

$$
F_{\lambda}^{j}\{\mathfrak{D}(\theta, K+2 p \bar{a} m)\} \subset \mathfrak{D}(\theta, K)=\mathfrak{D} .
$$

Our $K$ can be assumed to have been chosen so large that the succession of transformations (37), (41), (45), (47) maps $\mathfrak{D}(\theta, K)$ and $\mathfrak{D}(\theta, K+2 p \bar{a} m)$ one to one and conformally on to the domains $\mathbb{S}(j, \theta, K)$ and $\mathbb{S}(j, \theta, K+2 p \bar{a} m)$ respectively, defined after (50). Since by lemma 4 the functions $F_{\lambda}^{j}(t)$ and $f_{\lambda}(z)$ correspond under these transformations we have proved that $f_{\lambda}(z)$ is regular in $\mathscr{B}(j, \theta, K+2 p \bar{a} m)$ and that

$$
f_{\lambda}\{\leftrightarrow(j, \theta, K+2 p \bar{a} m)\} \subset \leftrightarrow(j, \theta, K) .
$$

Such a result holds for any $1 \leqq j \leqq m$ so that we may choose $K$ so large that (63) holds simultaneously for $j=1,2, \cdots m$. Then if $\subseteq(\theta, K)$ is the star (51) we have $f_{\lambda}(z)$ regular in $\subseteq(\theta, K+2 p \bar{a} m)$ and

$$
f_{\lambda}\{S(\theta, K+2 p \bar{a})\} \subset \subseteq(\theta, K) \text {. }
$$

The choice of $K$ depends in no way on the particular value of $\lambda$ chosen and these results hold uniformly for all $\lambda \epsilon \mathfrak{R}_{p}$.

We now remark that since $\lambda=1$ is in $\Re_{p}$, so also is $\lambda=-1$. Replacing $f(z)$ by 


$$
g(z)=f_{-1}(z)=z-a_{m+1} z^{m+1}+\cdots=z+a_{m+1}^{\prime} z^{m+1}+\cdots
$$

in the above discussion and noting that $g_{\lambda}=f_{-\lambda}$ so that the class $\Re_{p}$ is the same for both $f$ and $g$ one sees that the only change is in the parameter $\gamma$ of (41), (43), (50), which must now have such a value $\gamma^{\prime}$ that

$$
-a_{m+1}^{\prime} \exp \left(-i \gamma^{\prime} m\right)=a_{m+1} \exp \left(-i m \gamma^{\prime}\right)>0
$$

instead of

$$
-a_{m+1} \exp (-i m \gamma)>0 .
$$

It is legitimate to choose

$$
\gamma^{\prime}=\gamma+\pi / m
$$

The result (64) becomes in this case: There is a $K>0$ such that for any $\lambda \in \Re_{p}$ the function $g_{\lambda}(z)$ or $f_{\lambda}(z)$ (since $\lambda \in \Re_{p}$ implies $-\lambda \in \Re_{p}$ ) is regular in the star $\mathcal{S}^{\prime}(\theta, K+2 p \bar{a} m)$ and

$$
f_{\lambda}\left\{\Im^{\prime}(\theta, K+2 p a \bar{m})\right\} \subset S^{\prime}(\theta, K) .
$$

In this result the $\operatorname{star} \mathfrak{S}^{\prime}(\theta, K)$ is $\bigcup_{1}^{m} \mathcal{S S}^{\prime}(j, \theta, K)$ precisely as in (50), (51). Each domain $\mathscr{B S}^{\prime}(j, \theta, K)$ is a domain bounded by a simple closed curve analytic except at $z=0$ where two arcs meet in the directions

$$
\left\{\begin{array}{l}
\arg z=-\gamma+\frac{\theta}{m}-2 j \frac{\pi}{m}, \\
\arg z=-\gamma-\frac{\theta}{m}-(2 j-2) \frac{\pi}{m} .
\end{array}\right.
$$

In fact $\mathfrak{S}^{\prime}(\theta, K)$ has the appearance of $\mathfrak{S}(\theta, K)$ rotated through an angle of $-\pi / m$.

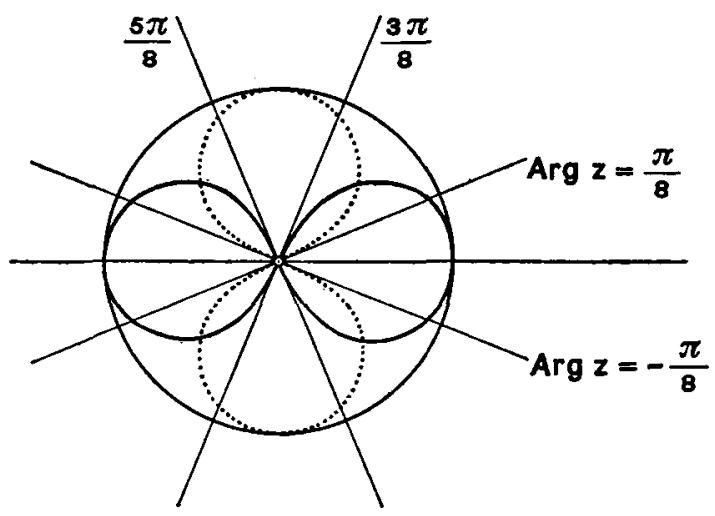

Fig. 4. 
We choose a $K$ valid both for (64) and (65). Since $0<\theta<\pi / 2$, the set

$$
\mathfrak{S}(\theta, K+2 p \bar{a} m) \cup \mathfrak{S}^{\prime}(\theta, K+2 p \bar{a} m) \cup\{z \mid z=0\}
$$

includes a circle $|z| \leqq \rho$ of positive radius. For every $\lambda \in \Omega_{p}$ the function $f_{\lambda}(z)$ is regular in this circle and for $|z| \leqq \rho$ one has by (64), (65)

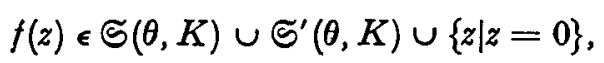

which is a bounded set. The theorem is proved. The case $m=2, \theta=\pi / 4$, $\gamma=0$ is illustrated in Fig. 4. The boundary of $\mathcal{S}^{\prime}(\theta, K)$ is dotted.

\section{Convergence of the family $t_{\lambda}$}

If $\Re$ is the set of $\lambda$ values corresponding to convergent members $f_{\lambda}$ of the family (7), our task is to describe the possible forms of $\Re$. Obviously $\Re$ satisfies the lattice condition that it $\lambda \epsilon \mathfrak{\Re}$ and $\mu \epsilon \Re$, then $m \lambda+n \mu \epsilon \mathfrak{R}$ for all positive or negative integers $m$ and $n$.

Lemma 6. The set $\Re$ defined above is closed if $\lambda=\infty$ be adjoined to it.

PROof: Consider any sequence $\left\{\lambda_{n}\right\}, \lambda_{n} \in \Re, n=1,2, \cdots$ which converges to a finite value $\mu$. All the $\lambda_{n}$ satisfy

$$
\left|\lambda_{n}\right| \leqq p, \lambda_{n} \in \mathbb{R}_{p}
$$

for some $p$ and by theorem 7 there is a circle $|z| \leqq \rho$ in which $\left\{f_{\lambda_{n}}\right\}$ form a uniformly bounded or normal family. We can therefore extract from $\left\{f_{\lambda_{n}}\right\}$ a subsequence, which we may as well assume to be $\left\{f_{\lambda_{n}}\right\}$ itself, and which converges uniformly to a limit $g(z)$ in $|z| \leqq \rho . g(z)$ is either the infinite constant or a regular function. But since $f_{\lambda_{n}}(0)=0$ it follows that $g(0)=0$ and in the same way

$$
g(z)=z+\mu a_{m+1} z^{m+1}+\cdots=f_{\mu}(z),
$$

since one has coefficientwise convergence also and the coefficients of $f_{\lambda}(z)$ are polynomials in $\lambda$. Thus the function $f_{\mu}(z)$ is convergent in $|z| \leqq \rho$ and $\mu \in \Re$.

From the lattice property of $\Re$ one sees that either every value of $\Re$ is a point of accumulation of $\Re$ or no finite point of $\Re$ is a point of accumulation. From this remark and lemma 6 follows

LEMma 7. If $\mathfrak{L}$ is any line through the origin of the complex $\lambda$-plane then $\mathfrak{\Omega} \cap \mathfrak{L}$ is either (i) the set $\{\lambda=0\}$, (ii) the set $\mathfrak{L}$ or (iii) a set of the form $\left\{n \lambda_{0}\right\}$, $n=0, \pm 1, \pm 2, \cdots$ where $\lambda_{0}$ is one of the two values of least nonzero modulus in $\mathfrak{R} \cap \mathbb{R}$. 
THEOREM 8. If the set $\Re$ corresponding to the convergent members of a family (7) contains a whole line $\mathfrak{Q}$ which passes through the origin, then $\mathbb{\Re}$ is the whole $\lambda$-plane.

In the proof of theorem 8 we use the following lemma.

LEMMA 8. If for the segment $z=x e^{i \theta},-1 \leqq x \leqq 1$, the polynomial $P_{n}(z)$ of degree $n$ satisfies

$$
\left|P_{n}(z)\right|<M,
$$

then for all complex $z$ such that $|z|<1$ holds, one has

$$
\left|P_{n}(z)\right|<M(\sqrt{ } 2+1)^{n}
$$

Proof: It suffices to take the case $\theta=0$. Then $z=\frac{1}{2}\left(t+t^{-1}\right)$ maps the circumference of the unit $t$-circle on to $-1 \leqq x \leqq 1$ in the $z$-plane. Thus

$$
\left|t^{n} P_{n}\left\{\frac{1}{2}\left(t+t^{-1}\right)\right\}\right|<M \text { for }|t|=1 .
$$

Every non-real point $z$ in $|z|<1$ has exactly one image point in $|t|<1$ and this image point $t$ has

$$
|t|>\sqrt{ } 2-1 .
$$

Thus for all $z$ in $|z| \leqq 1$ one has from (66) and the assumptions of the lemma:

$$
\left|P_{n}(z)\right|<M(\sqrt{ } 2+1)^{n} .
$$

ProOF OF THEOREM 8.

Take $p=1$. By theorem 7 there is a circle $|z| \leqq \rho$ in which all $f_{\lambda}(z)$ with $\lambda \in \mathfrak{\Re}_{p}$ are uniformly bounded by (say) $M>0$. In particular this is the case for all $\lambda \in \mathfrak{L} \cap \mathfrak{R}_{p}$. Hence the coefficients $b_{n}(\lambda)$ in (7) satisfy

$$
\left|b_{n}(\lambda)\right|<M \rho^{-n} \text { for } \lambda \in \mathfrak{Q} \cap \mathfrak{A}_{p}, n=2,3, \cdots
$$

By lemma 8 it follows that

$$
\left|b_{n}(\lambda)\right|<M \rho^{-n}(\sqrt{ } 2+1)^{n} \quad \text { for all } \quad|\lambda| \leqq p=1, n=2,3, \cdots
$$

Hence (7) converges for $|z|<\rho /(\sqrt{ } 2+1)$ for all $|\lambda| \leqq p$ and $\Re_{p}$ is the whole circle $|\lambda| \leqq p$, so that $\Omega$ is the whole plane.

A strengthening of theorem 8 is provided by

THEOREM 9: The set $\Re$ of theorem 8 has no finite points of accumulation unless it consists of the whole plane.

Proof. Take the case when $\mathfrak{R}$ is not the whole plane. We have to show there is no finite point of accumulation in $\Re$. By the lattice property of $\Re$ it is enough to show that $\lambda=0$ is not a point of accumulation. Suppose $\lambda=0$ is a point of accumulation. By theorem 8 any line $\mathfrak{Q}$ passing through $\lambda=0$ 
will not belong wholly to $\mathfrak{R}$ and by lemma 7 there is a minimal $|\lambda(\Omega)|$ for which $\lambda(\mathfrak{L}) \in \mathfrak{L} \cap \Re,|\lambda(\mathfrak{R})|>0$. This value may be infinite. By our assumption that 0 is a point of accumulation of $\Omega$ it follows that there is a sequence of lines $\mathfrak{L}(n), n=1,2, \cdots$ for which $\lambda(\mathfrak{L}(n)) \rightarrow 0$. We may without loss of generality assume that the lines $\mathfrak{L}(n)$ tend to a limit line $\mathfrak{R}$. For any point $\lambda_{1} \in \mathfrak{R}$ and $\varepsilon>0$ we may find a line $\mathfrak{R}(n)$ of the sequence such that $(a)$ the perpendicular distance from $\lambda_{1}$ to $\mathfrak{R}(n)$ is less than $\varepsilon / 2$ and (b) $|\lambda(\mathfrak{L}(n))|<\varepsilon / 2$. It follows that there is a member of $\Re$ (on $\mathfrak{L}(n)$ ) closer to $\lambda_{1}$ than $\varepsilon$. Thus $\lambda_{1}$ is in the closed set $\Re$ and since it is an arbitrary pointof $\Re$, the whole line $\Re$ is contained in $\mathfrak{Q}$. By theorem $8 \Re$ must be the whole plane. This contradiction shows that $\lambda=0$ is not a point of accumulation and the theorem is proved.

THEOREM 10. If $\AA$ is the set of $\lambda$ corresponding to the convergent members of the family (7) it has one of the forms

(i) the point $\lambda=0$,

(ii) $\left\{n \lambda_{0}\right\}, n=0, \pm 1, \pm 2, \cdots$ with $\lambda_{0} \neq 0$, a linear set,

(iii) $\left\{m \lambda_{0}+n \lambda_{1}\right\}, m=0_{1} \pm 1, \cdots, n=0, \pm 1, \cdots, \lambda_{0} / \lambda_{1}$ not real, $\lambda_{0} \neq 0_{1} \lambda_{1} \neq 0$, a plane lattice, or

(iv) the whole plane.

Proof: If (iv) does not apply the set $\Re$ is descrete. If $\Re$ contains points other than $\lambda=0$ we take a finite circle $|\lambda| \leqq M$ containing such points. $|\lambda| \leqq M$ contains at most a finite number of members of $\AA$ and we choose $\lambda_{0}$ to be one of these with minimal modulus (excluding $\lambda=0$ ). Then all points $n \lambda_{0}, n=0, \pm 1, \pm 2, \cdots$ lie in $\Re$ and if there are no others we have the case (ii). Certainly these are the only members of $\Re$ which lie on the line $\mathscr{R}$ : $\arg \lambda=\arg \lambda_{0}$. If $\Re$ contains further members take that one of smallest modulus which is first encountered on anticlockwise rotation of $\mathbb{R}$ and call it $\lambda_{1}$. Clearly $\lambda_{0} / \lambda_{1}$ is not real, $\lambda_{0} \neq 0, \lambda_{1} \neq 0$, and by a standard argument $\Omega$ is exhausted by $m \lambda_{0}+n \lambda_{1}, m=0, \pm 1, \pm 2, \cdots n=0, \pm 1, \pm 2, \cdots$ Every such point is a point of $₫$ and this is case (iv).

THEOREM 11. Cases (i), (ii) and (iv) of theorem 10 are realised.

We have not been able to find an example of case (iii).

Proof. (i). The sequences $\left\{a_{n}\right\}$ and $\left\{M_{n}\right\}, n=2,3, \cdots$ are constructed inductively as follows: $a_{2}=M_{2}=1$. If $a_{2}, \cdots, a_{n}$ and $M_{2}, \cdots M_{n}$ are already constructed set

$$
g(n, z)=z+a_{2} z^{2}+\cdots+a_{n} z^{n}
$$

and let $g_{\lambda}(n, z)$ be that series of the form

$$
g_{\lambda}(n, z)=z+\lambda a_{2} z^{2}+\sum_{m=3}^{n} c_{m}(\lambda) z^{m}+b_{n+1}(\lambda) z^{n+1}+\text { higher terms, }
$$


which commutes formally with $g(n, z)$. Choose

$$
M_{n+1}=\operatorname{Max}\left\{M_{n}, \operatorname{Max}_{|\lambda| \leq n}\left|b_{n+1}(\lambda)\right|\right\} .
$$

Then $M_{n+1} \geqq M_{n}$. Choose also

$$
a_{n+1}=M_{n+1}(n+1)^{n+1} .
$$

Then the series

$$
f_{1}(z)=z+\sum_{n=2}^{\infty} a_{n} z^{n}
$$

provides an example of case (1). In proof of this assertion: if

$$
f_{\lambda}(z)=z+\lambda a_{2} z^{2}+\sum_{3}^{\infty} A_{n}(\lambda) z^{n}
$$

is the commuting family of the form (7) containing (70), then calculation shows that

$$
A_{n+1}(\lambda)=\lambda a_{n+1}+b_{n+1}(\lambda) .
$$

For fixed $\lambda \neq 0$ we have for sufficiently large $n$

$$
\begin{aligned}
\left|\frac{A_{n+1}(\lambda)}{A_{n}(\lambda)}\right| & =\mid \frac{\lambda a_{n+1}+b_{n+1}(\lambda)}{\lambda a_{n}+b_{n}(\lambda)}>\frac{|\lambda| M_{n+1}(n+1)^{n+1}-M_{n+1}}{|\lambda| M_{n} n^{n}+M_{n}} \\
& >\frac{\lambda(n+1)^{n+1}-1}{\lambda n^{n}+1}
\end{aligned}
$$

by (69) and (68). This ratio tends to $\infty$ as $n \rightarrow \infty$ so that the series (71) diverges for every $\lambda \neq 0$. For $\lambda=0$ the series becomes identically $f_{0}(z) \equiv z$.

CASE (ii). In this part we consider the example provided by

$$
f_{1}(z)=e^{z}-1=z+\frac{1}{2} z^{2}+\cdots
$$

and the family (7):

$$
f_{\lambda}(z)=z+\frac{1}{2} \lambda z^{2}+\cdots
$$

generated by it. In $[1$, p. 160] it was shown that the only real values in $\Re$ are the integers. We show now that (72) cannot have a positive radius of convergence for any non-real $\lambda$. Suppose $\lambda$ is not real and that (72) converges in a circle $|z|\langle\rho, \rho\rangle 0$. In [1, p. 147 Satz 8] it was shown that the only nonconstant entire functions which commute with $f_{1}(z)=e^{z}-1$ are the natural iterates $f_{n}(z), n=0,1,2, \cdots$ Thus $f_{\lambda}(z)$ is not entire and $\rho<\infty$. The process used in [1, pp. 161--2, Satz 17 parts (i), (ii) and (iii)] shows that $f_{\lambda}(z)$ has no singularity in the plane cut along the infinite segment 
$-\infty<\operatorname{Re} z \leqq-\rho$. There must be at least one singularity of $f_{\lambda}(z)$ on the circumference $|z|=\rho$ and this must occur at $z=-\rho$.

If on $-\rho<x<0$ the function $f_{\lambda}(z)$ takes any real value, say if $f_{\lambda}\left(x_{1}\right)$ is real for $-\rho<x_{1}<0$, it follows from $0>f_{1}(x)>x, x<0$, that $f_{\lambda}\left(f_{1}(x)\right)=f_{1}\left(f_{\lambda}(x)\right)$ holds on $\left[x_{1}, 0\right]$. Then $f_{\lambda}\left(f_{1}\left(x_{1}\right)\right)=f_{1}\left(f_{\lambda}\left(x_{1}\right)\right)$ is a real quantity. Thus if $x_{2}=f\left(x_{1}\right)$ we have $0>x_{2}>x_{1}$ and $f_{\lambda}\left(x_{2}\right)$ real. Defining $x_{n}=f\left(x_{n-1}\right)$ for $n=2,3, \cdots$ we have $0>x_{n}>x_{n-1}$ and $f_{\lambda}\left(x_{n}\right)$ real. There is a number $l$ such that $x_{n} \rightarrow l \leqq 0$ as $n \rightarrow \infty$ and one must have $f_{1}(l)=l$ so that $l=0$. But since

$$
f_{\lambda}(x)=x+\frac{\lambda x^{2}}{2}+\cdots, \lambda \text { not real, }
$$

it is impossible that $f_{\lambda}\left(x_{n}\right)$ be real for $x_{n} \rightarrow 0$. Consequently $f_{\lambda}(z)$ takes no real value in $(-\rho, 0)$.

Since for $x<0$ one has $f_{1}(x)>x$, it is possible to choose $\rho_{1}>\rho$ such that $f_{1}\left(-\rho_{1}\right)>-\rho$. Then $f_{\lambda}\left(f_{1}(x)\right.$ is regular for $-\rho_{1}<x<0$ and takes no real values there. Therefore $f_{-1}\left(f_{\lambda}\left(f_{1}(x)\right)\right)$ is regular on $-\rho_{1}<x<0$ and gives a continuation of $f_{\lambda}(x)$ there, which is regular over $z=-\rho$. This contradicts the fact that $z=-\rho$ is a singularity of $f_{\lambda}(z)$, so that the assumption $\rho>0$ is false.

CASE (iv). This is realised by the family

$$
f_{\lambda}(z)=\frac{z}{1-\lambda z}=\sum_{n=0}^{\infty} \lambda^{n} z^{n+1}
$$

which forms a continuous group of substitutions.

\section{Existence of an infinitesimal transformation}

In case (iv) of theorems 10 and 11 one has a local continuous group of functions $f_{\lambda}(z)$ with complex parameter $\lambda$ and

$$
f_{\lambda}\left(f_{\mu}(z)\right)=f_{\lambda+\mu}(z) \text {. }
$$

The individual transformations have expansions of the form (7). If we restrict $\lambda$ to $|\lambda| \leqq 1$, there is a $\delta>0$ such that all these $f_{\lambda}(z)$ converge in $|z| \leqq \delta$, where they also satisfy $|f(z)|<M$ for a suitable constant $M$. The coefficients in (7) satisfy

$$
\left|b_{n}(\lambda)\right| \leqq M \delta^{-n} \text { for }|\lambda| \leqq 1 .
$$

Now for $n \geqq m+1$ we have shown in theorem 2 that $b_{n}(\lambda)$ is polynomial degree at most $(n-m)$. From (12) $b_{n}(0)=0$. By the lemma of Schwarz 


$$
\left|\frac{b_{n}(\lambda)}{\lambda}\right| \leqq M 8^{-n} \text { for }|\lambda| \leqq 1
$$

and

$$
\left|b_{n}^{\prime}(0)\right| \leqq M \delta^{-n} .
$$

Also

$$
p_{n}(\lambda)=\frac{b_{n}(\lambda)}{\lambda}-b_{n}^{\prime}(0)
$$

is a polynomial in $\lambda$ whose constant term vanishes.

$$
\left|p_{n}(\lambda)\right| \leqq\left|b_{n}(\lambda)\right|+\left|b_{n}^{\prime}(0)\right| \leqq 2 M \delta^{-n} \text { for }|\lambda|=1 \text {, }
$$

and since $p_{n}(0)=0$ one has

$$
\left|p_{n}(\lambda)\right| \leqq 2 M \delta^{-n}|\lambda| \text { for }|\lambda| \leqq 1 .
$$

The series

$$
I(z)=a_{m+1} z^{m+1}+\sum_{n=m+2}^{\infty} b_{n}^{\prime}(0) z^{n}
$$

converges for $|z|<\delta$, where moreover

$$
\begin{aligned}
\left|I(z)-\left(\frac{f_{\lambda}(z)-z}{\lambda}\right)\right| & =\left|\sum_{n=m+1}^{\infty} p_{n}(\lambda) z^{n}\right| \\
& <|\lambda| \sum_{m+2}^{\infty} 2 M|z|^{n} \delta^{-n}=\frac{|\lambda|}{\delta^{m+1}} \cdot \frac{2 M|z|^{m+2}}{(\delta-|z|)}
\end{aligned}
$$

from (73). Thus

$$
I\langle z\rangle=\lim _{\lambda \rightarrow 0} \frac{f_{\lambda}(z)-z}{\lambda}
$$

holds for $|z|<\delta$ and $I(z)$ is the infinitesimal transformation of the group (7). The function $f_{\mu}(z)$ may be substituted in place of $z$ in (75), provided we make $\delta$ smaller, and (75) becomes

$$
I\left(f_{\mu}(z)\right)=\frac{\partial f_{\mu}(z)}{\partial \mu} .
$$

The existence of the derivative (76) shows that in a region of the form $|z|<\delta,|\mu|<p$ the function $f_{\mu}(z)$ is analytic in both $\mu$ and $z$. Taking a fixed $z \neq 0$ in $|z|<\delta$ and letting $\mu$ grow from 0 to $\lambda$ along the path $\mu=t \lambda$, $0 \leqq t \leqq 1$ we find that $f_{\mu}(z)$ traces a curve $\Gamma$ in $|z|<\delta$. If $\delta$ and $\lambda$ are sufficiently small this curve $\Gamma$ is simple and does not enclose the origin. Integrating (76) along this curve 


$$
\int_{\mu=0}^{\mu-\lambda} \frac{d f_{\mu}}{I\left(f_{\mu}\right)}=\lambda
$$

with the boundary conditions $f_{\mu}(z)=z$ when $\mu=0$ and $f_{\lambda}(z)$ when $\mu=\lambda$ we obtain

$$
A_{1}\left(f_{\lambda}(z)=A_{1}(z)+\lambda\right.
$$

where

$$
\begin{aligned}
& A_{1}(z)=\int^{z} \frac{d z}{I(z)} \text { has an expansion of the form } \\
& A_{1}(z)=\sum_{n=-m}^{-1} c_{n} z^{-n}+c_{0} \log z+\sum_{n=1}^{\infty} c_{n} z^{n} .
\end{aligned}
$$

Such a formula (77) is of the same type as (23) and (61). A function $A_{1}(z)$ satisfying (77) is called an Abel function and the equation itself Abel's functional equation. We see that there is an Abel function intimately connected with the infinitesimal transformation $I(z)$ of the group (7) and that this functional equation can be used to define $f_{\lambda}(z)$ at least in partial neighbourhoods of $z=0$. On the other hand the logarithmic term in (78) will in general be present and the Abel function will not be single valued around $z=0$. In general then one can not expect to study the continuous iteration near a fixpoint of multiplier 1 by finding an Abel function regular up to poles at $z=0$. A case where the function $A_{1}(z)$ is, however, single valued at $z=0$ is provided by the family

$$
f_{\lambda}(z)=\frac{z}{1-\lambda z}
$$

for which $I(z)=z^{2}$ and $A_{1}(z)=-z^{-1}$.

One may remark that from an Abel function of the form (78) or an infinitessimal transformation (74) one can construct a group (7). If for example the convergent series (74) is given, one has only to solve the equation (76), that is the differential equation

$$
\frac{d w}{d \mu}=I(w)
$$

with boundary condition $w=z$ at $\mu=0$. The solution $w(\mu, z)$ is analytic in both $\mu$ and $z$ in a neighbourhood of $\mu=0, z=0$ (e.g. Bieberbach [3]).

The function $w(\mu, z)$ has an expansion in terms of $z$ of the form

We put

$$
w(\mu, z)=z+\mu a_{m+1} z^{m+1}+\cdots
$$

$$
\begin{aligned}
& \chi_{1}=w\{\mu, w(\lambda, z)\} \\
& \chi_{2}=w\{\mu+\lambda, z\} .
\end{aligned}
$$


Then $\partial \chi_{1} / \partial \mu=I\left(\chi_{1}\right)$ and $\partial \chi_{2} / \partial \mu=I\left(\chi_{2}\right)$. These are simply equation (79) and are to be integrated with the boundary condition $\chi_{1}=\chi_{2}=v(\lambda, z)$ when $\mu=0$. Thus $\chi_{1} \equiv \chi_{2}$ and the functions $w(\mu, z)$ form a commuting group of series (7) whose infinitesimal transformation is $I(z)$.

In the cases (i), (ii) and (iii) where the family (7) does not converge for all $\lambda$, one may form the series (74) corresponding to $I(z)$ but the work of the preceding part of this paragraph shows that the series will not converge. For convergent series $I(z)$ correspond to case (iv) only. In the cases (ii) and (iii) it may be shown by further analysis that there is indeed a family of continuous iterates $f_{\lambda}(\dot{z})$ satisfying $f_{\lambda}\left(f_{\mu}\right)=f_{\mu}\left(f_{\lambda}\right)$ but most of these have a singularity at the origin. G. Szekeres $[15]$ has investigated such situations.

\section{Some applications to permutable entire functions}

In $\S 2$, theorem 6 , a simple application of theorems $1-5$ was made to the theory of entire functions. In the second example of theorem 11 the entire function $e^{z}-1$ occurred. It is natural then to investigate the case when certain members of a commuting family (7) are entire functions. We prove first the following

THEOREM 12. If $\left\{f_{\lambda}(z)\right\}$ is a commuting family (7), then the numbers $\lambda$ such that $f_{\lambda}(z)$ is entire form a discrete and hence countable set.

LEMMA 9. If the series

$$
f_{1}(z)=z+a_{m+1} z^{m+1}+\sum_{n=m+2}^{\infty} a_{n} z^{n}
$$

has a positive radius of convergence, then for given $\theta$ with $0<\theta<\pi / 2$ and all sufficiently large $K$ there is a star $\subseteq(\theta, K)$ of the form (51) such that

$$
\begin{gathered}
f_{1}\{\subseteq(\theta, K)\} \subset \subseteq(\theta, K), \\
f_{n}(z) \rightarrow 0 \text { in } \subseteq(\theta, K) \text { for all } z \in \subseteq(\theta, K) .
\end{gathered}
$$

Further there is a star $\mathbb{S}^{\prime}(\theta, K)$ of the form $\left(50^{\prime}\right)$, (51) (ct. proof of theorem 7 ) such that

$$
\begin{gathered}
f_{-1}\left\{\mathcal{S}^{\prime}(\theta, K)\right\} \subset \mathcal{S}^{\prime}(\theta, K), \\
f_{-n}(z) \rightarrow 0 \text { in } \mathcal{S}^{\prime}(\theta, K) \text { for all } z \in \mathcal{S}^{\prime}(\theta, K) .
\end{gathered}
$$

Proof: This follows immediately from the last part of lemma 4 with $\lambda=1$ together with the fact that, in the notation of lemma 4:

$$
F_{n}^{j}(z) \rightarrow \infty \text { as } n \rightarrow \infty \text { for any } z \in \mathscr{D}(\theta, K) \text { by (21). }
$$

We remark that since $K$ may be taken arbitrarily large, the regions $S(\theta, K)$ and $\mathfrak{S}^{\prime}(\theta, K)$ may be assumed interior to any given circle centred at $z=0$. 
Proof of Theorem 12. There is nothing to prove except when case (iv) of theorem 10 applies - and when for some $\lambda=0$ the function $f_{\lambda}(z)$ of $(7)$ is entire. There is no loss of generality in assuming that for $\lambda=1$ the function $f_{1}(z)$ is entire. Applying lemma 9 to $f_{1}(z)$ we form $\mathfrak{S}=\mathfrak{S}(\theta, K)$ and $\mathfrak{S}^{\prime}=$ $\mathfrak{S}^{\prime}(\theta, K)$ and note that $\mathfrak{S} \cup \mathfrak{S}^{\prime}$ may be supposed interior to a given circle $|z|\langle t, t>0$. According to theorem 7 there is to any $p>1$ a constant $\rho>0$ and a constant $M>0$ such that for all $|\lambda|<p$ the series $f_{\lambda}(z)$ converges in $|z| \leqq \rho$ and $\left|f_{\lambda}(z)\right| \leqq M$ for $|z| \leqq \rho$. Then

$$
\left|f_{\lambda}(z)\right| \leqq \frac{M|z|}{\rho} \text { for }|z| \leqq \rho .
$$

Choose the above $t$ so that $0<t \leqq \rho$ and so that for $|z|<t$ and $|\lambda| \leqq p$, the value $f_{\lambda}(z)$ is contained in a region where $f(z)$ is univalent. This is possible because $\left|f_{\lambda}(z)\right|<M t / \rho$.

Now, as was pointed out in the poof of theorem 7 (cf. Fig. 4), the domain $\mathfrak{S} \cup \mathfrak{S}^{\prime}$ contains a circle $|z|\langle\sigma, \sigma\rangle 0$. Since $f_{1}(z)=z+a_{m+1} z^{m+1}+\cdots$ holds, the sequence $\left\{f_{n}(z)\right\}$ is not normal near $z=0$. From Fatou [6], [5], $z=0$ is a point of accumulation of fixpoints of iterates $f_{n}(z)$. Thus there exist a point $\xi \neq 0$ and an integer $N>1$ such that

$$
|\xi|<\sigma, \quad \xi \in \subseteq \cup \mathcal{S}^{\prime}, \quad f_{N}(\xi)=\xi, \quad f_{3}(\xi) \neq \xi \text { for } j<N .
$$

Now for $n=1,2, \cdots$ one has $f_{n}(\xi)=f_{j}(\xi)$ where $1 \leqq j \leqq N$ and $j \equiv n$ (modulo $N$ ). Moreover $f_{j}(\xi) \neq f_{k}(\xi)$ for $1 \leqq j<k \leqq N$. Thus the sequence $\left\{f_{n}(\xi)\right\}$ does not converge to 0 . By (80) and (81) $\xi ; \mathbb{S}$. Thus by (84) $\xi \in \mathbb{S}^{\prime}$.

Suppose that there is an infinity of values $\lambda$ in $|\lambda| \leqq p$ for which $f_{\lambda}(z)$ is entire. These values have a point of accumulation $\mu,|\mu| \leqq p$. There is a sequence of values $\lambda_{n}, n=1,2, \cdots$ in which $\lambda_{m} \neq \lambda_{n}$ for $m \neq n$, such that $\lambda_{n} \rightarrow \mu$ and $f_{\lambda_{n}}(\xi) \rightarrow f_{\mu}(\xi)$ as $n \rightarrow \infty$. The values $f_{\lambda_{n}}(\xi)$ are fixpoints of $f_{N}(z)$ and thus members of a discrete set. Hence from a certain $n$ onwards all $f_{\lambda_{n}}(\xi)$ are the same. Thus if $\alpha=\lambda_{n}$ and $\beta=\lambda_{n+1} \neq \alpha$ are suitable members of the sequence we have $f_{\alpha}(\xi)=f_{\beta}(\xi)$.

Define inductively $\xi_{1}=\xi, \xi_{n+1}=f_{-1}\left(\xi_{n}\right)$ where $f_{-1}$ may be taken to be the series expansion $f_{-1}(z)=z-a_{m+1} z^{m+1}+\cdots$ and by lemma 9 the uniquely defined points $\xi_{n}$ lie in $\mathcal{S}^{\prime}$ for $n=1,2,3, \cdots$ As $n \rightarrow \infty$ we have $\xi_{n} \rightarrow 0$. We assert that $f_{\alpha}\left(\xi_{n}\right)=f_{\beta}\left(\xi_{n}\right)$ for all $n$. This is certainly true for $n=1$. Suppose the result holds for $n=1,2, \cdots, m-1$. Then

$$
f_{1}\left\{f_{\alpha}\left(\xi_{m}\right)\right\}=f_{\alpha}\left\{f\left(\xi_{m}\right)\right\}=f_{\alpha}\left(\xi_{m-1}\right)=f_{\beta}\left(\xi_{m-1}\right)=f_{1}\left\{f_{\beta}\left(\xi_{m}\right)\right\} \text {. }
$$

But $f_{\alpha}\left(\xi_{m}\right)$ and $f_{\beta}\left(\xi_{m}\right)$ lie in a region where $f(z)$ is univalent and so must be equal. Our assertion is proved by induction.

Since $f_{\alpha}\left(\xi_{n}\right)=f_{\beta}\left(\xi_{n}\right)$ and $\xi_{n} \rightarrow 0$ as $n \rightarrow \infty$ it follows that $f_{\alpha}(z) \equiv f_{\beta}(z)$ which can only be true if $\lambda_{n}=\alpha=\beta=\lambda_{n+1}$. This is a contradiction and it 
follows that our hypothesis of an infinite set of $\lambda$ with $|\lambda| \leqq p$ and $f_{\lambda}$ entire must be false. Since this applies for every $p>1$ the proof of theorem 12 is complete.

We have also shown that the entire members of a family (7) form at most a countable set. This set always contains the linear polynomial $f_{0}(z)=z$ and no other linear polynomial. There may be also a polynomial of degree two or more among the functions $f_{\lambda}(z)$ and in this case the family can include no transcendantal entire function. Conversely if there is a transcendental member of the family (7) then the only polynomial member is $f_{0}(z)=z$. These assertions follow from a result of Baker [1, p. 141] and Ganapathy Iyer [8]: no entire transcendental function can commute with a polynomial of degree two or more.

We now combine theorems 6 and 12 to obtain

THEOREM 13. If $F(z)$ is a non-linear entire function exactly one of rohose fixpoints has multiplier 1 , then the entire functions permutable with $F(z)$ form a countable set.

Proof: As in theorem 6 we may assume the fixpoint to be $z=0$ so that $F(z)$ has an expansion

$$
F(z)=z=a_{m+1} z^{m+1}+\sum_{n=m+2}^{\infty} a_{n} z^{n}, m \geqq 1, a_{m+1} \neq 0 .
$$

The constant functions permutable with $F(z)$ are the solutions $c$ of $F(c)=c$ which form at most a countable set. The non-constant functions permutable with $F(z)$ are either members of the family $(7): F_{\lambda}(z)$ of theorem 2 or by theorem 4 they have the form $H \circ F_{\lambda}(z)$ where $H_{m}(z) \equiv z$. The entire functions of the form $F_{\lambda}(z)$ form at most a countable set. If $H \circ F_{\lambda}(z)$ is entire then so is its $m$-th interate $F_{m \lambda}(z)$ which is in (7). Thus the entire functions of this second kind are also at most countable and the theorem is proved.

One may ask whether the set of entire functions permutable with a given entire function is countable under more general hypotheses then those of theorem 13. This is indeed so but other methods of proof are required.

Finally we prove

\section{TheOREM 14. If the members}

$$
f_{1}(z)=z+a_{m+1} z^{m+1}+\sum_{m+2}^{\infty} b_{n}(1) z^{n}, a_{m+1} \neq 0, m \geqq 1,
$$

and

$$
f_{\lambda}(z)=z+\lambda a_{m+1} z^{m+1}+\sum_{m+2}^{\infty} b_{n}(\lambda) z^{n}
$$

of the family (7) are entire, then $\lambda>0$. 
Proof: Suppose $\lambda=l e^{i \alpha}, l>0, \alpha \neq 0,-\pi<\alpha \leqq \pi$. Choose $\theta$ with $0<2 \theta<|\alpha|$. By lemma 9 there is a star $\mathfrak{S}=\mathfrak{S}(\theta, K)$ of the form (51) such that $f_{1}(\mathfrak{S}) \subset \mathbb{S}$ and $f_{n}(z) \rightarrow 0$ in $\mathbb{S}$ for $z \in \mathbb{S}$. By (50) the $m$ branches of the star $\mathbb{S}$ lie one in each of the angles

$$
\begin{aligned}
&-\gamma+\frac{\theta}{m}-(2 j-3) \frac{\pi}{m}<\arg z<-\gamma-\frac{\theta}{m}-(2 j-1) \frac{\pi}{m}, \\
& j=1,2, \cdots, m,
\end{aligned}
$$

i.e. in angles of magnitude $2(\pi-\theta) / m$ spaced evenly around $z=0$ with a gap of $2 \theta / \mathrm{m}$ between neighbouring angles. The boundary of $\mathfrak{S}$ is tangent at $z=0$ to the arms of the angles (85). By (43) the term $\gamma$ in (85) is determined by $-a_{m+1} \exp (-i \gamma m)>0$.

Applying the same reasoning to the function $f_{\lambda}(z)$ we see that there is a star $\Im=\Im(\theta, K)$ of the form (51) such that $f_{\lambda}(\Im) \subset \Im$ and $f_{n \lambda}(z) \rightarrow 0$ in $\Im$ for $z \in \Im$. The $m$ branches of $\Im$ lie one in each of the angles

$$
\begin{aligned}
-\gamma^{\prime}+\frac{\theta}{m}-(2 j-3) \frac{\pi}{m} & <\arg z<-\gamma^{\prime}-\frac{\theta}{m}-(2 j-1) \frac{\pi}{m}, \\
j & =1,2, \cdots m,
\end{aligned}
$$

where by (43) $\gamma^{\prime}$ is determined by

$$
-\lambda a_{m+1} \exp \left(-i \gamma^{\prime} m\right)>0 \text {. }
$$

Since $\lambda=l e^{i \alpha}, l>0$, we may put $\gamma^{\prime}=\gamma+\alpha / m$. Thus by (85), (86) the

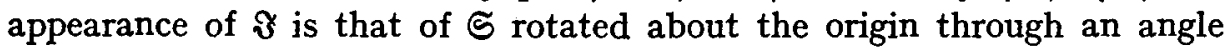
$-\alpha / m$. Since $2 \theta / m<|\alpha| / m<2(\pi-\theta) / m$, it follows that $\subseteq \cup \Im$ contains a circle $|z|\langle r, r\rangle 0$. We remark further that since the $K$ in $\subseteq(\theta, K)$ and $\Im(\theta, K)$ may be arbitrarily enlarged, the region $\varsigma \cup \Im$ may be assumed to be contained in an arbitrarily small circle centred at the origin.

By the results of Baker [2] or Rosenbloom [13], since $f_{1}(z)$ is a nonlinear entire function, it follows that for all but at most one of the values $j=2$, 3,4 the equation $f_{j}(z)=z$ has a solution $\xi$ which is not a solution of $f_{k}(z)=z$ for $1 \leqq k<j$. Thus there are points $\xi$ and $\eta$ with $f(\xi) \neq \xi, f(\eta) \neq \eta, \xi \neq \eta$, $f_{12}(\xi)=\xi, f_{12}(\eta)=\eta$. Clearly $\xi \neq 0, \eta \neq 0$. By the above remark we may suppose $\mathfrak{S} \cup \Im$ so close to $z=0$ that $\xi \notin \subseteq \cup \Im, \eta \notin \subseteq \cup \Im$ Now since

$$
f_{n \lambda}(z)=z+n \lambda a_{m+1} z^{m+1}+\cdots, n=1,2, \cdots
$$

it follows that the sequence $\left\{f_{n \lambda}\right\}$ is not normal in $|z|<r$ and there is a value $\left|z_{1}\right|<r$ and an integer $N \geqq 1$ such that

$$
f_{N \lambda}\left(z_{1}\right)=\xi \text { or } t_{N \lambda}\left(z_{1}\right)=\eta
$$


Suppose $f_{N \lambda}\left(z_{1}\right)=\xi$, which is no loss of generality. Then since $f_{12}(\xi)=\xi$, we have for any positive integer $m$

$$
f_{12 m}\left\{f_{N \lambda}\left(z_{1}\right)\right\}=\xi, m=1,2,3, \cdots
$$

If $z_{1} \in \subseteq$ then $f_{12 m}\left(z_{1}\right) \rightarrow 0$ as $m \rightarrow \infty$ and hence $f_{N \lambda}\left\{f_{12 m}\left(z_{1}\right)\right\} \rightarrow 0$. Therefore

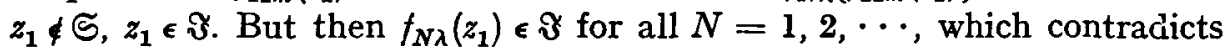
$f_{N \lambda}\left(z_{1}\right)=\xi \notin \mathfrak{\Im} \cup \mathfrak{S}$. Thus the assumption $\alpha \neq 0$ is false.

\section{References}

[1] Baker, I. N., Zusammensetzungen ganzer Funktionen. Math. Z. 69, 121-163 (1958).

[2] Baker, I. N., The existence of fixpoints of entire functions. Math. Z. 73, 280-284 (1960).

[3] Bieberbach, L., Theorie der gewöhnlichen Differentialgleichungen. Berlin: Springer, 1953.

[4] Cremer, H., Über die Häufigkeit der Nichtzentren. Math. Ann. 115, 673-580 (1938).

[5] Fatou, P., Sur les équations fonctionelles. Bull. Soc. Math. France 47, 161-271 (1919); 48, 33-94, 208-314 (1920).

[6] Fatou, P., Sur l'itération des fonctions transcendantes entières. Acta Math. 47, 337 -370 (1926).

[7] Hadamard, J., Two works on iteration and related questions. Bull. Amer. Math. Soc. 50, $67-75(1944)$.

[8] Ganapathy Iyer, V., On permutable integral functions. J. Lond. Math. Soc. 34, 141 - 144 (1959).

[9] Jacobsthal, E., Uber vertauschbare Polynome. Math. Z. 63, 243-276 (1955).

[10] Julia, G., Mémoire sur la permutabilité des fractions rationelles. Annales sci. de l'École Normale Superieure (3) 39, $131-215$ (1922).

[11] Koenigs, G., Recherches sur les intégrales de certaines équations fonctionelles. Annales sci. de l'École Normale Supérieure (3), Suppl., (1884), 3-41.

[12] Ritt, J. F., Prime and composite polynomials. Trans. Amer. Math. Soc. 23, $51-66$ (1922).

[13] Rosenbloom, P., The fix-points of entire functions. Medd. Lunds Univ. mat. Sem., suppl. Bd. M. Riesz 186-192 (1952).

[14] Siegel, C. L., Iteration of analytic functions. Annals Math. 43, 607-616 (1942).

[15] Szekeres, G., Regular iteration of real and complex functions. Acta Math. 100, 203-258 (1958).

Imperial College of Science and Technology London. 